\title{
Estudo comparativo do perfil metabólico e antropométrico de pacientes com a síndrome dos ovários policísticos em tratamento com anticoncepcionais orais contendo drospirenona ou ciproterona
}

\author{
Comparative study of the metabolic and anthropometric profile of patients with polycystic ovary \\ syndrome under treatment with oral contraceptives containing drospirenone or cyproterone
}

Estudio comparativo del perfil metabólico y antropométrico de pacientes con síndrome de ovario poliquístico en tratamiento con anticonceptivos orales que contienen drospirenona o ciproterona

\section{Resumo}

A Síndrome dos Ovários Policísticos (SOP) é uma disfunção endócrina heterogênea. Caracteriza-se por oligoamenorreia e hiperandrogenismo. Associa-se a comorbidades como resistência insulínica, hiperlipidemia, doenças cardiovasculares, problemas cosméticos e infertilidade. Objetivo: Analisar e comparar possíveis impactos nos perfis antropométrico, metabólico e cardiovascular de pacientes com SOP antes e após 06 meses de tratamento com anticoncepcional combinado oral (ACO), contendo $0,035 \mathrm{mg}$ de Etinilestradiol (EE) + 2mg de Ciproterona ou 0,03mg de EE + 3mg de Drospirenona. Metodologia: Estudo observacional, prospectivo e analítico, com abordagem quantitativa, aprovado pelo CEP do HU-UFPI, parecer n2.568.977, realizado entre agosto/2018-junho/2019. Selecionou-se mulheres de 18 a 40 anos com diagnóstico confirmado para SOP, divididas em dois grupos proporcionais para uso das seguintes combinações, por 06 meses: Grupo 1 (EE + Ciproterona) e Grupo 2 (EE + Drospirenona). Utilizou-se os testes estatísticos t-Student e U de Mann-Whitney para análise dos dados, nível de 5\% de significância. Resultados: Analisou-se os dados antropométricos e metabólicos das 16 participantes selecionadas antes e após 6 meses de terapia com ACO. Os dois grupos foram homogêneos no início da pesquisa. Houve redução estatisticamente significativa do IMC no grupo 2 e aumento significativo do HDL e do Colesterol Total no grupo 1. Conclusão: Após 06 meses de uso desses ACO, observou-se: melhora do colesterol total e HDL com EE+Ciproterona; redução do IMC com EE + Drospirenona; ausência de risco adicional no perfil metabólico, antropométrico e cardiovascular e de superioridade clínica no risco metabólico de uma combinação hormonal sobre a outra em pacientes com SOP.

Palavras-chave: Síndrome dos ovários policísticos; Síndrome metabólica; Anticoncepcional oral.

\begin{abstract}
Abstrast
Polycystic Ovary Syndrome (PCOS) is a heterogeneous endocrine dysfunction characterized by oligoamenorrhea and hyperandrogenism. It is associated with comorbidities such as insulin resistance, hyperlipidemia, cardiovascular diseases, cosmetic problems and infertility. Analyse and compare possible impacts on the anthropometric, metabolic and cardiovascular profiles of patients with PCOS before and after 06 months of treatment with combined oral contraceptives (COC) containing Ethinylestradiol (EE) 0.035mg + Cyproterone 2mg or EE 0.03mg + Drospirenone
\end{abstract}


3mg. Observational, prospective and analytical study, with quantitative approach, approved by the REC of HU-UFPI (number 2.568.977), accomplished between August 2018-June 2019. Women aged 18 to 40 years with a confirmed diagnosis for PCOS were selected and divided into two homogeneous groups to use the following combinations for 06 months: Group 1 (EE + Cyproterone) and Group 2 (EE + Drospirenone). Statistical t-Student and Mann-Whitney $\mathrm{U}$ tests were used for data analysis, with a 5\% significance level. Anthropometric and metabolic data of the 16 selected participants were analyzed before and after 6 months of COC therapy. There was a statistically significant reduction in BMI in group 2 and a significant increase in HDL and Total Cholesterol in group 1. After 06 months of using these COCs, it was observed: improvement in Total Cholesterol and HDL with EE + Cyproterone; reduction in BMI with EE + Drospirenone; absence of additional risk in the metabolic, anthropometric and cardiovascular profile; no clinical superiority in the metabolic risk of one hormonal combination over the other in patients with PCOS.

Keywords: Polycystic ovary syndrome; Metabolic syndrome; Oral contraceptive.

\section{Resumen}

El síndrome de ovario poliquístico (SOP) es una disfunción endocrina heterogénea caracterizada por oligoamenorrea y hiperandrogenismo. Se asocia a comorbilidades como resistencia a la insulina, hiperlipidemia, enfermedades cardiovasculares, problemas cosméticos y infertilidad. Analizar y comparar posibles impactos en los perfiles antropométricos, metabólicos y cardiovasculares de pacientes con SOP antes y después de 06 meses de tratamiento con anticonceptivos orales combinados (AOC) que contienen etinilestradiol (EE) 0,035 mg + ciproterona $2 \mathrm{mg}$ o EE $0,03 \mathrm{mg}+$ drospirenona $3 \mathrm{mg}$. Estudio observacional, prospectivo y analítico, con enfoque cuantitativo, aprobado por el REC de HU-UFPI (número 2.568.977), realizado entre agosto 2018-junio 2019. Se seleccionaron y dividieron mujeres de 18 a 40 años con diagnóstico confirmado de SOP en dos grupos homogéneos para usar las siguientes combinaciones durante 06 meses: Grupo 1 (EE + Ciproterona) y Grupo 2 (EE + Drospirenona). Para el análisis de los datos se utilizaron las pruebas estadísticas t-Student y Mann-Whitney U, con un nivel de significancia del 5\%. Se analizaron los datos antropométricos y metabólicos de los 16 participantes seleccionados antes y después de 6 meses de tratamiento con AOC. Hubo una reducción estadísticamente significativa en el IMC en el grupo 2 y un aumento significativo en HDL y colesterol total en el grupo. Después de 06 meses de uso de estos AOC, se observó: mejoría del Colesterol Total y HDL con EE + Ciproterona; reducción del IMC con EE + Drospirenona; ausencia de riesgo adicional en el perfil metabólico, antropométrico y cardiovascular; sin superioridad clínica en el riesgo metabólico de una combinación hormonal sobre la otra en pacientes con SOP.

Palabras clave: Síndrome de ovario poliquístico; Síndrome metabólico; Anticonceptivo oral.

\section{Introdução}

A Síndrome dos Ovários Policísticos (SOP) é uma disfunção endócrina heterogênea com taxa de prevalência que varia de 13,9\% a 15\% das mulheres em idade reprodutiva (Finotti, 2013). Esta síndrome foi primeiramente descrita em 1935 por Stein e Leventhal e possui como quadro clínico amenorreia ou oligomenorreia, devido a um quadro crônico de ciclos anovulatórios, associado a um hiperandrogenismo. Consequentemente, esta síndrome pode provocar inúmeras comorbidades que repercutem na saúde física e mental das mulheres acometidas. Dentre essas comorbidades, destacam-se a resistência insulínica, hiperlipidemia, doenças cardiovasculares, problemas cosméticos (hirsutismo, acne vulgaris e obesidade, calvície androgenética) e infertilidade. Segundo o Consenso de Roterdam (2003), o diagnóstico de SOP é dado quando 2 dos 3 critérios estiverem presentes: 1) Anovulação crônica; 2) Hiperandrogenismo clínico e/ou laboratorial; 3) Ovário policístico visualizado através de ultrassonografia (Brandão et al., 2005). Além disso, é necessário excluir outras possíveis causas de hiperandrogenismo (Brandao et al., 2005; Christ et al., 2014; Lopes et al., 2011).

A etiologia desta síndrome ainda não foi bem definida, no entanto, admite-se haver influência de fatores genéticos com modulação ambiental (Brandao et al., 2005; Christ et al., 2014; Hong et al., 2017). Outro mecanismo associado a patogênese da SOP é a hiperinsulinemia, o que foi descrito em 1921 por Archad e Thiers (Christ et al., 2014). Devido ao aumento de insulina plasmática, este hormônio passa a ligar-se mais avidamente nos receptores de insulina no ovário, estimulando a ação da enzima 17-hidroxilase, a qual promove o aumento da produção androgênica. Além disso, a insulina 
inibe a produção hepática da globulina ligadora de hormônios sexuais (SHBG), o que provoca o aumento da fração livre de testosterona (hormônio ativo) (Brandão et al., 2005). A produção excessiva de androgênios provoca a hiperplasia folicular que causa espessamento e atresia dos folículos, gerando assim o aspecto policístico do ovário à ultrassonografia (Brandão et al., 2005; Jeanes; Reeves, 2017).

O tratamento da SOP varia de acordo com os principais sintomas e comorbidades presentes em cada paciente assim como o desejo de engravidar. O tratamento de primeira linha inclui medidas comportamentais (orientação dietética e atividade física em pacientes obesas, resistentes à insulina e dislipidêmicas por exemplo) e o uso de contraceptivos hormonais combinados (ACO) (Melo et al., 2017). A utilização dos ACOs suprime a liberação excessiva de LH e consequentemente a produção demasiada de androgênios. Eles também causam uma competição antagônica com a testosterona nos receptores (promovido pelo componente progestagênico do ACO), assim como o aumento da produção da SHBG (promovido pelo componente estrogênico). Dessa forma, o ACO promove a regulação do ciclo menstrual, evita o sangramento uterino disfuncional e melhora o quadro hiperandrogênico (Abeso, 2010). Além disso, o ACO diminui os riscos de hiperplasia e adenocarcinomas endometriais por evitar a exposição ao excesso de progesterona e estrogênios no endométrio (Brandão, et al., 2005).

Os ACO contém um componente estrogênico (Etinilestradiol, valerato de estradiol ou estradiol) e um componente progestagênio que varia em termo de composição e afinidade aos receptores de outros hormônios esteroides (mineralocorticoides, glicocorticoides, androgênico e estrogênico). Tanto o estrogênio quanto o progestagênio contribuem para o manejo das manifestações clínicas (Melo et al., 2017, Ehrman, 2005, Nader 2007).

A utilização de ACO, por outro lado, pode causar mudanças nos perfis lipídicos das pacientes e na pressão arterial (Guastella; Longo; Carmina, 2010; Hong, S, et al, 2017). Pode provocar também alterações no perfil metabólico, antropométrico e endócrino, além de resistência à insulina, em pacientes com SOP, ainda não bem definidas, e os estudos apontam resultados conflitantes (Mes-Krowinkel, 2014). Portanto foi desenhado um estudo para pesquisar quais seriam as possíveis diferenças no perfil metabólico das mulheres que utilizam ACO combinados com componente progestagênios com efeitos antiandrogênicos para tratamento de SOP em um contexto local e quais fatores influenciariam nessas diferenças.

O presente estudo tem como objetivo principal analisar os possíveis impactos nos perfis antropométricos, metabólicos e saúde cardiovascular de pacientes com síndrome dos ovários policísticos (SOP) antes e após 06 meses de tratamento com anticoncepcional hormonal oral (ACO), contendo 0,035mg de Etinilestradiol (EE) $+2 \mathrm{mg}$ de Ciproterona ou 0,03mg de de EE + 3mg de Drospirenona, em mulheres com SOP, Além de comparar o perfil antropométrico, metabólico (mediante avaliação de lipidograma, HOMA IR, insulina basal e TOTG 2h) e saúde cardiovascular em pacientes com SOP antes e após 06 meses de tratamento com ACO contendo 0,035mg de Etinilestradiol (EE) + 2mg de Ciproterona, buscando também comparar o perfil antropométrico, metabólico (mediante avaliação de lipidograma, HOMA IR, insulina basal e TOTG 2h) e saúde cardiovascular em pacientes com SOP antes e após 06 meses de tratamento com ACO contendo 0,03mg de Etinilestradiol (EE) + 3 mg Drospirenona, e por fim comparar o perfil antropométrico, metabólico e saúde cardiovascular entre pacientes com SOP antes e após 06 meses de tratamento com ACO contendo 0,035mg de Etinilestradiol (EE) + 2mg de Ciproterona e pacientes com SOP antes e após tratamento com ACO contendo 0,03mg de Etinilestradiol (EE) + $3 \mathrm{mg}$ Drospirenona.

\section{Metodologia}

Trata-se de um estudo observacional, prospectivo e analítico, com abordagem quantitativa, realizado no setor de ginecologia do Hospital Universitário (HU) da Universidade Federal do Piaú, no período compreendido entre agosto de 2018 a junho de 2019. 
Foram selecionadas 40 participantes, tendo como critérios de inclusão a idade de 18 a 40 anos, com diagnóstico confirmado para SOP segundo os critérios diagnósticos de Roterdam (2012) (presença de dois dos três critérios: anovulação crônica; hiperandrogenismo clínico e/ou laboratorial e ovários policísticos na ultrassonografia pélvica/transvaginal, excluindo-se outras causas de hiperandrogenismo) e que procuraram atendimento médico para contracepção. Todas as participantes assinaram os Termos de Consentimento Livre e Esclarecido, conforme a Res. 466/2006 e Complementares, do Conselho Nacional de Saúde (CNS).

As participantes foram divididas em dois grupos: Grupo 1: 20 mulheres, que usariam Anticoncepcional hormonal oral combinado (ACO) contendo 0,035mg de Etinilestradiol (EE) + 2mg de Ciproterona (CIP), e Grupo 2: 20 mulheres que usariam Anticoncepcional hormonal Oral combinado (ACO) contendo 0,03mg de de EE $+3 \mathrm{mg}$ de Drospirenona (DRS). A todas as pacientes com suspeita clínica de SOP foram solicitados os exames complementares: FSH/LH, S-DHEA, 17-OH-P, TSH e T4 livre; Insulina basal; TOTG (75g) de 2h; Colesterol total, LDL-c; HDL-c; Triglicerídeos; Androstenediona, Testosterona total e livre; Glicemia de jejum; Prolactina; HOMA-IR; US transvaginal ou US pélvica, antes do início e após 6 meses do uso dos contraceptivos.

Foram excluídas 13 participantes dos dois grupos pela realização dos exames complementares incompletos. Posteriormente, mais 11 participantes foram excluídas por descontinuarem o tratamento, não retornarem à consulta com os exames de controle após 6 meses de terapia ou apresentarem desfechos diversos, como a decisão de engravidar, totalizando um espaço amostral de n=16 participantes, 9 no grupo 1 (EE+CIP) e 7 no grupo 2 (EE+DRS).

A fim de investigar as diferenças entre os grupos estudados, no período inicial e após 6 meses, foi utilizado o teste estatístico $t$-Student com o nível de 5\% de significância estabelecido no estudo. Todas as análises foram realizadas levando-se em conta o tipo de estudo e com auxílio do software R, versão 3.6.0, utilizando bibliotecas padrões do programa. Para comparação entre os grupos (intergrupo) foi utilizado o teste U de Mann-Whitney. Em todos os testes foram empregados 0,05 de nível de significância, ou seja, quando valor de $p$ for menor que 0,05 há diferença estatisticamente significante.

\subsection{Aspectos éticos e legais}

A pesquisa em questão foi previamente autorizada pela instituição a ser pesquisada (CAPP-HU-UFPI) e submetida e aprovada pelo Comitê de Ética em Pesquisa (CEP) do Hospital Universitário da Universidade Federal do Piauí, HU-UFPI, número do parecer 2.568.977 obedecendo às normatizações da resolução 466/2012 do CNS.

Todas as participantes do estudo assinaram o Termo de Consentimento Livre e Esclarecido (TCLE).

Como benefícios da pesquisa pode-se citar a detecção de síndrome dos ovários policísticos e do risco metabólico associado. Além da contribuição para o conhecimento do tema e para formação de base de dados.

Não há benefício extra nesta pesquisa no que se refere ao tratamento e/ou planejamento familiar, porém os benefícios se estendem a comunidade científica e a possibilidade de os resultados da pesquisa serem úteis na evolução e escolha do contraceptivo no tratamento das mulheres com Síndrome de Ovários Policísticos, além de permitir a instituição precoce da terapêutica multidisciplinar para obtenção de melhores resultados ginecológicos, endócrinos e cardiovasculares a longo prazo. Para a participante da pesquisa terá uma possibilidade de um acompanhamento aprimorado do uso dos contraceptivos, com a resolução imediata dos possíveis efeitos adversos.

\section{Resultados}

Foram analisados os dados antropométricos e metabólicos das 16 participantes do estudo antes do início da terapia com ACO e após 6 meses de uso contínuo. 
Conforme evidenciado na Tabela 1, os grupos 1 (EE+CIP) e 2 (EE+DRS) foram homogêneos, não apresentando diferenças significativas desde o início da pesquisa, com exceção da idade, com Grupo 1 apresentando média de idade de 25,8 e o Grupo 2 de 31,7 anos, o que não interfere nos resultados finais da pesquisa.

Tabela 1: Perfil clínico das pacientes com SOP antes do início da terapia com ACO.

\begin{tabular}{|c|c|c|c|}
\hline & \multicolumn{2}{|c|}{ Média ( \pm dp) } & \multirow{2}{*}{ Valor } \\
\hline & G1 & G2 & \\
\hline Idade (anos) & $25,8( \pm 5,5)$ & $31,7( \pm 5,2)$ & 0,0442 \\
\hline IMC & $27,2( \pm 4,8)$ & $25,2( \pm 4,2)$ & 0,3823 \\
\hline $\mathrm{FSH}(\mathrm{mU} / \mathrm{mL})$ & $4,2( \pm 1,2)$ & $3,7( \pm 1,6)$ & 0,4604 \\
\hline LH (mU/mL) & $11,0( \pm 7,7)$ & $7,4( \pm 4,6)$ & 0,2702 \\
\hline Testosterona Total (ng/dL) & $34,0( \pm 63,1)$ & $29,2( \pm 21,5)$ & 0,8384 \\
\hline Testosterona Livre (ng/dL) & $2,0( \pm 2,8)$ & $0,8( \pm 0,4)$ & 0,2537 \\
\hline 17 hidroxiprogesterona (ng/dL) & $118,1( \pm 22,7)$ & $\begin{array}{c}134,3 \\
( \pm 42,9)\end{array}$ & 0,3894 \\
\hline SDHEA $(\mathrm{mg} / \mathrm{mL})$ & $\begin{array}{c}366,2 \\
( \pm 514,8)\end{array}$ & $\begin{array}{c}161,7 \\
( \pm 39,3)\end{array}$ & 0,2687 \\
\hline Androstenediona (ng/ml) & $3,7( \pm 1,2)$ & $2,6( \pm 1,1)$ & 0,0770 \\
\hline Prolactina $(\mathrm{ng} / \mathrm{mL})$ & $13,2( \pm 6,5)$ & $15,9( \pm 5,3)$ & 0,3800 \\
\hline TSH (microUI/mL) & $1,9( \pm 1,3)$ & $1,9( \pm 1,0)$ & 0,8851 \\
\hline T4-livre (ng/mL) & $1,0( \pm 0,2)$ & $1,0( \pm 0,1)$ & 0,7528 \\
\hline Glicemia jejum (mg/dL) & $77,1( \pm 9,4)$ & $82,0( \pm 8,5)$ & 0,2947 \\
\hline Insulina basal (microUI/mL) & $10,7( \pm 4,7)$ & $12,5( \pm 7,7)$ & 0,5933 \\
\hline Triglicerídeos (mg/dL) & $110,3( \pm 56,8)$ & $\begin{array}{c}126,6 \\
( \pm 60,4)\end{array}$ & 0,5921 \\
\hline $\mathrm{LDL}(\mathrm{mg} / \mathrm{dL})$ & $91,1( \pm 21,0)$ & $99,1( \pm 37,6)$ & 0,6238 \\
\hline HDL (mg/dL) & $45,6( \pm 12,5)$ & $60,3( \pm 19,1)$ & 0,1096 \\
\hline Colesterol Total (mg/dL) & $162,6( \pm 31,6)$ & $\begin{array}{c}180,7 \\
( \pm 31,4)\end{array}$ & 0,2723 \\
\hline HOMA IR & $1,9( \pm 0,9)$ & $2,5( \pm 1,6)$ & 0,3815 \\
\hline Circunferencia abdominal $(\mathrm{cm})$ & $80,2( \pm 13,9)$ & $81,9( \pm 10,3)$ & 0,7906 \\
\hline Circunferencia do quadril $(\mathrm{cm})$ & $106,2( \pm 13,0)$ & $99,6( \pm 8,2)$ & 0,2323 \\
\hline Pressão sistólica (mmHg) & $113,3( \pm 10,0)$ & $\begin{array}{c}111,4 \\
( \pm 10,7) \\
\end{array}$ & 0,7222 \\
\hline Pressão diastólica (mmHg) & $76,7( \pm 8,7)$ & $77,1( \pm 9,5)$ & 0,9194 \\
\hline
\end{tabular}

${ }^{*}$ Teste $t$ para diferença de médias entre as variáveis do grupo.

Legenda: G1 - Grupo 1 (EE + CIP); G2 - Grupo 2 (EE+DRS).

Fonte: Pesquisa direta no ambulatório de Saúde da Mulher - HU-UFPI.

As Tabelas 2 e 3 evidenciam as mudanças nos perfis antropométricos e metabólicos das pacientes em uso de EE+CIP e EE+DRS por 6 meses, respectivamente. Quanto aos dados antropométricos, o IMC sofreu leve acréscimo no grupo 1, assim como as circunferências abdominal, enquanto a circunferência do quadril se manteve praticamente sem alterações, contudo não estatisticamente significante. No grupo 2 , o IMC sofreu redução estatisticamente significante $(25,2 \pm 4,2$; $24,5 \pm 4,2$ ) enquanto as circunferências abdominal e do quadril sofreram mudanças não significativas. Em ambos os grupos, a PA não sofreu alterações importantes. 
Quanto aos dados metabólicos, observou-se que houve um aumento significativo nos valores de HDL nas pacientes do grupo $1(45,6 \pm 12,5 ; 56,6 \pm 12,0)$, assim como do colesterol total $(162,6 \pm 31,6 ; 174,4 \pm 22,4)$. Os níveis de triglicerídeos também aumentaram, enquanto o LDL sofreu redução, porém, ambos não significativos estatisticamente. No grupo 2, houve acréscimo nos níveis de HDL e triglicerídeos, entretanto, não foram estatisticamente significativos. Diferentemente do grupo 1, no grupo 2 houve discreto aumento nos níveis de LDL e redução do CT.

Tabela 2: Perfil antropométrico e metabólico antes e após 6 meses de uso de EE+CIP em pacientes com SOP.

\begin{tabular}{c|c|c|c}
\hline \multirow{2}{*}{} & \multicolumn{2}{|c|}{ Média $( \pm \mathbf{d p})$} & \multirow{2}{*}{ Valor $p$} \\
\cline { 2 - 3 } & Início & $\mathbf{6}$ meses & \\
\hline IMC & $27,2( \pm 4,8)$ & $28,0( \pm 5,3)$ & 0,3299 \\
\hline Glicemia jejum $(\mathrm{mg} / \mathrm{dL})$ & $77,1( \pm 9,4)$ & $74,7( \pm 11,5)$ & 0,6087 \\
\hline Insulina basal $(\mathrm{microUI} / \mathrm{mL})$ & $10,7( \pm 4,7)$ & $17,7( \pm 18,9)$ & 0,2766 \\
\hline Triglicerídeos $(\mathrm{mg} / \mathrm{dL})$ & $110,3( \pm 56,8)$ & $150,2( \pm 54,6)$ & 0,0753 \\
\hline LDL $(\mathrm{mg} / \mathrm{dL})$ & $91,1( \pm 21,0)$ & $87,9( \pm 15,8)$ & 0,6300 \\
\hline HDL $(\mathrm{mg} / \mathrm{dL})$ & $45,6( \pm 12,5)$ & $56,6( \pm 12,0)$ & $0,0116^{*}$ \\
\hline Colesterol Total $(\mathrm{mg} / \mathrm{dL})$ & $162,6( \pm 31,6)$ & $174,4( \pm 22,4)$ & $0,0441^{*}$ \\
\hline HOMA IR & $1,9( \pm 0,9)$ & $4,2( \pm 4,1)$ & 0,0927 \\
\hline Circunferencia abdominal $(\mathrm{cm})$ & $80,2( \pm 13,9)$ & $80,8( \pm 13,5)$ & 0,4560 \\
\hline Circunferencia do quadril $(\mathrm{cm})$ & $106,2( \pm 13,0)$ & $106,1( \pm 10,0)$ & 0,9332 \\
\hline Pressão sistólica $(\mathrm{mmHg})$ & $113,3( \pm 10,0)$ & $113,3( \pm 8,7)$ & 1,0000 \\
\hline Pressão diastólica $(\mathrm{mmHg})$ & $76,7( \pm 8,7)$ & $81,1( \pm 6,0)$ & 0,1038 \\
\hline
\end{tabular}

*Teste $t$ para diferença de médias entre as variáveis do grupo. Fonte: Pesquisa direta no ambulatório de Saúde da Mulher - HU UFPI.

Tabela 3: Perfil antropométrico e metabólico antes e após 6 meses de uso de EE+DRS em pacientes com SOP.

\begin{tabular}{c|c|c|c}
\hline \multirow{2}{*}{} & \multicolumn{2}{|c|}{ Média $( \pm \mathbf{d p})$} & \multirow{2}{*}{ Valor $p$} \\
\cline { 2 - 3 } & Início & $\mathbf{6}$ meses & \\
\hline IMC & $25,2( \pm 4,2)$ & $24,5( \pm 4,2)$ & $0,0407^{*}$ \\
\hline Glicemia jejum $(\mathrm{mg} / \mathrm{dL})$ & $82,0( \pm 8,5)$ & $76,7( \pm 8,0)$ & 0,1414 \\
\hline Insulina basal $(\mathrm{microUI} / \mathrm{mL})$ & $12,5( \pm 7,7)$ & $11,6( \pm 6,1)$ & 0,4963 \\
\hline Triglicerídeos $(\mathrm{mg} / \mathrm{dL})$ & $126,6( \pm 60,4)$ & $189,7( \pm 87,5)$ & 0,1534 \\
\hline LDL $(\mathrm{mg} / \mathrm{dL})$ & $99,1( \pm 37,6)$ & $108,4( \pm 50,7)$ & 0,5600 \\
\hline HDL $(\mathrm{mg} / \mathrm{dL})$ & $60,3( \pm 19,1)$ & $68,9( \pm 17,8)$ & 0,2618 \\
\hline Colesterol Total $(\mathrm{mg} / \mathrm{dL})$ & $180,7( \pm 31,4)$ & $179,6( \pm 43,0)$ & 0,9483 \\
\hline HOMA IR & $2,5( \pm 1,6)$ & $3,3( \pm 2,4)$ & 0,5184 \\
\hline Circunferencia abdominal $(\mathrm{cm})$ & $81,9( \pm 10,3)$ & $81,4( \pm 9,3)$ & 0,7541 \\
\hline Circunferencia do quadril $(\mathrm{cm})$ & $99,6( \pm 8,2)$ & $101,9( \pm 7,1)$ & 0,4376 \\
\hline Pressão sistólica $(\mathrm{mmHg})$ & $111,4( \pm 10,7)$ & $112,9( \pm 11,1)$ & 0,8182 \\
\hline Pressão diastólica $(\mathrm{mmHg})$ & $77,1( \pm 9,5)$ & $75,7( \pm 11,3)$ & 0,7882 \\
\hline
\end{tabular}

${ }^{*}$ Teste $t$ para diferença de médias entre as variáveis do grupo Fonte: Pesquisa direta no ambulatório de Saúde da Mulher - HU UFPI. 
Com relação ao metabolismo dos carboidratos, o Grupo 1 apresentou redução da Glicemia de Jejum e aumento da Insulina Basal e do HOMA IR, porém nenhum resultado estatisticamente significativo. No grupo 2, apenas o HOMA IR sofreu acréscimo, havendo reduções não significativas nos níveis de Glicemia de Jejum e Insulina Basal.

A Tabela 4 é referente à média dos valores do TOTG 75g 2h após ingestão de glicose nos Grupos 1 e 2 antes e 6 meses após o início da terapia com ACO. No grupo 1, a média dos valores de TOTG 75g 2h após ingestão de glicose sofreu uma redução não significativa estatisticamente $(111,6 \pm 39,23 ; 102,4 \pm 26,1)$ após 6 meses de uso do ACO. No grupo 2, houve um aumento também não estatisticamente significante dos valores de TOTG após os 6 meses de uso do ACO contendo EE+DRS.

Tabela 4: Média dos valores de TOTG 75g 2h após ingestão de glicose, antes e após 6 meses de uso de EE+CIP e EE +DRS, em pacientes com SOP.

\begin{tabular}{cccc}
\hline & \multicolumn{2}{c}{ Média $( \pm \mathrm{DP})$} & \multirow{2}{*}{ p-valor* } \\
\cline { 1 - 3 } & Início & $\mathbf{6}$ meses & \\
\hline Grupo 1 (EE+CIP) & $111,6( \pm 39,23)$ & $102,4( \pm 26,1)$ & 0,5712 \\
\hline Grupo 1 (EE+DRS) & $99,7( \pm 21,4)$ & $104,3( \pm 28,0)$ & 0,7376 \\
\hline
\end{tabular}

*Teste $t$ de diferença de médias entre os grupos

Fonte: Pesquisa direta no ambulatório de Saúde da Mulher - HU UFPI.

Na Tabela 5, tem-se as medianas (IIQ) das diferenças entre início e após 6 meses de tratamento por variável e grupo, a fim de verificar diferença entre a variação dos tratamentos dos grupos CIP e DRS no tempo. No que diz respeito à essas diferenças das variações dos tratamentos em cada variável e grupo, pode-se constatar que não houve diferença significativa entre os grupos (valor de $p>0,05$ ).

Tabela 5: Medianas (IIQ) das diferenças entre após 6 meses e início do tratamento por grupo.

\begin{tabular}{|c|c|c|c|}
\hline \multirow{2}{*}{ Variáveis } & \multicolumn{2}{|c|}{ Grupos } & \multirow[b]{2}{*}{ Valor de $p$} \\
\hline & CIP & DRS & \\
\hline IMC & $0,53(1,18)$ & $-0,46(1,59)$ & 0,071 \\
\hline Circunferência abdominal (cm) & $0(2)$ & $-1(4)$ & 0,252 \\
\hline Circunferência do quadril (cm) & $1(2)$ & $-2(12)$ & 0,681 \\
\hline Pressão sistólica (mmHg) & $40(10)$ & $30(20)$ & 0,918 \\
\hline Pressão diastólica (mmHg) & $0(10)$ & $0(20)$ & 0,299 \\
\hline Glicemia jejum (mg/dL) & $-3(16)$ & $-2(13)$ & 0,758 \\
\hline Insulina basal (microUI/mL) & $1,66(9,35)$ & $0,2(3,7)$ & 0,299 \\
\hline Triglicerídeos (mg/dL) & $26(110)$ & $82(176)$ & 0,681 \\
\hline $\mathrm{LDL}(\mathrm{mg} / \mathrm{dL})$ & $-0,6(8)$ & $1,6(43,1)$ & 0,470 \\
\hline $\mathrm{HDL}(\mathrm{mg} / \mathrm{dL})$ & $9(20)$ & $8(30)$ & 0,837 \\
\hline Colesterol Total (mg/dL) & $10(15)$ & $10,3(76)$ & 0,918 \\
\hline HOMA IR & $0,32(2,26)$ & $-0,09(2,19)$ & 0,174 \\
\hline TOTG (mg/dl) & $0(10)$ & $5(26)$ & 0,252 \\
\hline
\end{tabular}

* Teste U de Mann-Whitney. IIQ = intervalo interquartil Fonte: Pesquisa Direta no ambulatório de Saúde da Mulher - HU UFPI. 


\section{Discussão}

A Síndrome dos Ovários Policísticos pode provocar inúmeras comorbidades que repercutem na saúde física e mental das mulheres acometidas, devido ao quadro crônico de ciclos anovulatórios associado ao hiperandrogenismo característico. Dentre essas comorbidades, destacam-se a resistência insulínica, hiperlipidemia, doenças cardiovasculares, problemas cosméticos (hirsutismo, acne vulgaris e obesidade, calvície androgenética) e infertilidade (Teed et al., 2018). O manejo clínico de primeira linha da SOP, especialmente da irregularidade menstrual, do hirsutismo e da acne, inclui mudança de estilo de vida e uso de ACO, os quais podem impactar no perfil metabólico e cardiovascular de suas usuárias (Melo et al., 2017).

Estudos já publicados, com alto nível de evidência, foram capazes de demonstrar os benefícios da terapia com contraceptivos orais combinados em pacientes com SOP, principalmente nas pacientes com perfil hiperandrogênico, visto que a maior parte das preparações reduz os androgênios e aumenta os níveis de globulina ligadora de hormônios sexuais, no que diz respeito especialmente aos sinais e sintomas de hiperandroginismo cosméticos e de irregularidade menstrual. Com relação ao impacto no perfil metabólico, faltam estudos que permitam fortes recomendações baseadas em evidência.

Uma combinação ideal de ACO ainda não foi estabelecida. Uma revisão sistemática de 42 estudos experimentais com metanálise demonstrou que independentemente do tipo de progestágeno presente no contraceptivo oral combinado (COCs) contendo 20-35 micrograma de EE, o uso de COCs foi associado com uma redução de $61 \%$ nos níveis de testosterona livre. COCs contendo acetato de ciproterona parecem apresentar maior atividade antiandrogênica que desogestrel e drospirenona em usuários de longo prazo (12 meses ou mais), mas não em curto/médio prazo (até 6 meses) (Melo et al., 2017).

Nesse estudo foram analisados os dados antropométricos e metabólicos das 16 participantes do estudo antes do início da terapia com ACO e após 6 meses de uso contínuo. Os grupos 1 e 2 foram homogêneos, não apresentando diferenças significativas desde o início da pesquisa.

Obesidade abdominal e aumento da ativação do sistema inflamatório são observados em pacientes com peso normal e obesos com SOP, levando a um risco aumentado de dislipidemia, diabetes e possivelmente doença cardiovascular. Pacientes com diagnóstico de SOP, portanto, devem ser rastreados para elementos da síndrome metabólica, incluindo peso, cintura, pressão arterial HbA1c e status lipídico (Glintborg, 2016). Evidências de pesquisas específicas sobre a associação entre contraceptivos hormonais combinados e peso corporal em mulheres com SOP parecem estar completamente ausentes, mas muitos estudos investigaram os efeitos de diferentes anticoncepcionais hormonais sobre o peso corporal em mulheres saudáveis (Melo et al., 2017). Contudo, ainda não se tem evidência suficiente para determinar com segurança qual seria esse efeito, sendo necessários estudos com grupo controle ou não-hormonal, para controle de outros possíveis fatores que afetem no peso (Gallo et al., 2014).

No presente estudo, verificou-se que não ocorreram alterações significativas nos parâmetros antropométricos IMC e circunferencias abdominal e do quadril após o uso de ACO durante seis meses pelo Grupo 1 (EE + CIP), enquanto o Grupo 2 $(E E+D R S)$ apresentou redução significativa no IMC das pacientes com SOP (Tabela 03). Em outro estudo desenvolvido por Aydin, et al. com o objetivo de avaliar a influência da combinação etinilestradiol e drospirenona na composição corporal de mulheres com SOP, observou-se que o tratamento oral combinado por seis meses em pacientes com SOP resultou em um aumento da porcentagem de gordura total e do tronco, apesar de nenhuma mudança nas medidas antropométricas clínicas, incluindo peso, IMC e relação cintura-quadril (Aydin et al., 2013). Estudos mais aprofundados e com amostras maiores são necessários para verificar o efeito dos contraceptivos orais combinados sobre os dados antropométricos de mulheres com SOP, especialmente em mulheres não obesas antes do início do tratamento. 
Recentemente, muitos estudos vêm sendo feitos com o intuito de analisar o impacto no perfil metabólico e risco cardiovascular em pacientes com SOP em uso de ACO. Hipertensão arterial sistólica é considerado um efeito adverso de ACO, assim como pior controle da PA em mulheres previamente hipertensas (Medeiros, 2017). Entretanto, há estudos mostrando que não há aumento significativo na pressão arterial em pacientes com SOP em tratamento com ACO (Melo et al., 2017). O estudo aqui apresentado também não mostrou diferenças significativas na PA, sistólica ou diastólica, para ambos os grupos (Tabelas 02 e 03).

A SOP é fator de risco para Resistência à insulina, Diabetes Mellitus tipo 2 e Diabetes Gestacional, independentemente de idade e/ou obesidade. No que tange ao metabolismo dos carboidratos, ACO têm sido associados com aumento da glicemia de jejum, insulina basal e TOTG em pacientes sem a síndrome dos ovários policísticos, mas os resultados são inconsistentes. Quanto aos resultados existentes para pacientes com SOP, ainda não se pode concluir o efeito clínico real dos ACO com relação ao metabolismo de carboidratos, mas, aparentemente, não acrescentam nenhum risco adicional, tanto para a combinação com ciproterona quanto drospirenona. Com relação ao HOMA-IR, a combinação de EE+CIP pode ser usada e até mesmo melhorar a resistência à insulina dos usuários; com relação à drospirenona, os dados são inconsistentes (Melo et al., 2017). Uma revisão sistemática e metanálise de 31 ensaios clínicos avaliou a participação dos ACO no metabolismo dos carboidratos em mulheres sem Diabetes Mellitus tipo 2 e demostrou que, independentemente da via de administração e do tipo de estrogênio (EE ou natural), os ACOs têm efeito limitado no metabolismo de carboidratos, e, consequentemente, não tem efeito adverso adicional para a saúde endócrina (Lopez; Grimes; Schulz, 2014).

Em conformidade com a literatura, no estudo em questão observou-se que não houve mudança estatisticamente significativa nos valores de glicemia de jejum, insulina basal, HOMA-IR e no TOTG 2h, antes e após 6 meses de uso de ACO, em ambos os grupos (Tabelas 02, 03 e 04). Deve- se levar em consideração que outras intervenções afetam essa relação, como: mudanças de estilo de vida, uso de metiformina, dentre outras. O guideline atual baseado em evidência científica de baixa-moderada qualidade recomenda mudança do estilo de vida em pacientes com SOP e excesso de peso, visando à redução de peso, obesidade central e resistência à insulina, bem como considerar o uso de metformina (Teed, 2018).

Quanto ao perfil lipídico, a maioria dos ACO aumenta consistentemente HDL, CT e triglicerídeos, mas para se determinar os reais impactos clínicos dessa mudança mais estudos, em especial à longo prazo, são necessários (Medeiros, 2017). Os resultados encontrados no estudo em questão fortalecem a associação entre o uso de ACO e alterações no perfil lipídico, visto que houve aumento estatisticamente significativo de HDL (bom colesterol) e CT em um dos grupos avaliados (grupo 1: EE + CIP). Entretanto, a observação por apenas 6 meses e o tamanho amostral não permitem tirar conclusões à respeito do real impacto cardiovascular desse achado.

As alterações relativas a todos os parâmetros antropométricos e metabólicos estudados após seis meses de tratamento com ACO, em comparação com os níveis basais em ambos os grupos são mostradas na Tabela 05. No presente estudo, embora o uso de ACO contendo 0,035mg de Etinilestradiol (EE) + $2 \mathrm{mg}$ de Ciproterona por seis meses tenha aumentado os níveis de CT e HDL e o uso de ACO contendo 0,03mg de Etinilestradiol (EE) + 3 mg Drospirenona por seis meses tenha reduzido o IMC das pacientes com SOP, os impactos dessas duas formulações sobre o metabolismo, antropometria e saúde cardiovascular se mostraram similares, não sendo verificada diferença estatisticamente significativa entre os dois grupos em nenhum dos parâmetros utilizados. Portanto, não foi observada superioridade clínica de um ACO sobre o outro. Outros estudos envolvendo amostras maiores e períodos de acompanhamento mais prolongados são necessários para elucidar integralmente os efeitos de cada um desses ACOs sobre os perfis metabólicos, antropometria e saúde cardiovascular de pacientes com SOP. 


\section{Conclusão}

Como conclusão desse estudo pode-se observar que mulheres com SOP em tratamento com ACO contendo Etinilestradiol+ ciproterona melhoraram os níveis de colesterol total e HDL após 06 meses de tratamento. O uso da preparação de ACO contendo Etinilestradiol + drospirenona na SOP reduziu o IMC após 06 meses de tratamento.

Os contraceptivos hormonais combinados orais contendo etinilestradiol $(0,35 \mathrm{mg})$ associado a ciproterona (2mg) parece não ter impacto geral sobre o IMC, Cintura abdominal, Cintura do quadril, Pressão arterial, Glicemia de jejum, HOMA-IR, Insulina basal, Colesterol total, LDL e HDL das mulheres com SOP quando comparado com os contraceptivos combinados contendo Etinilestradiol (0,30mg) e Drospirenona (3mg) durante 6 meses, corroborando com a hipótese de que o uso de anticoncepcionais hormonais orais combinados nessas dosagens não causa danos adicionais no perfil metabólico e antropométrico e risco cardiovascular dessas mulheres.

Não há superioridade clínica significativa quanto ao risco metabólico entre o uso em pacientes com SOP da preparação de etinilestradiol $(0,35 \mathrm{mg})+$ ciproterona $(2 \mathrm{mg})$ sobre a preparação de etinilestradiol $(0,3 \mathrm{mg})+$ drospirenona (3mg), e vice-versa.

\section{Referências}

Abeso. (2009). Associação Brasileira para o estudo da obesidade e da Síndrome Metabólica. ABESO $3^{a}$ ed. São Paulo, 2010.

Brandão, A.P. et al. (2005). I Diretriz brasileira de diagnóstico e tratamento da síndrome metabólica. Arq Bras Cardiol, 84, 1-28

Aydin, K., Cinar, N., Aksoy, D. Y., Bozdag, G., \& Yildiz, B. O. (2013). Body composition in lean women with polycystic ovary syndrome: effect of ethinyl estradiol and drospirenone combination. Contraception, 87(3), 358-362.

Brasil. Ministério da Saúde. Consulta Pública no 33, de 26 de agosto de 2010. Brasília: Secretaria de Atenção à Saúde, 2010. http://bvsms.saude.gov.br/bvs/saudelegis/sas/2010/cop0033_26_08_2010.html.

Christ, J. P., Willis, A. D., Brooks, E. D., Brink, H. V., Jarrett, B. Y., Pierson, R. A., ... \& Lujan, M. E. (2014). Follicle number, not assessments of the ovarian stroma, represents the best ultrasonographic marker of polycystic ovary syndrome. Fertility and sterility, 101(1), $280-287$.

Finotti, M. Repercussões não reprodutivas da SOP na saúde da mulher. (20130. O que e como avaliar? In: congresso de ginecologia e obstetrícia do distrito federal, 46. Brasília: SGOB.

Gallo, M. F., Lopez, L. M., Grimes, D. A., Carayon, F., Schulz, K. F., \& Helmerhorst, F. M. (2014). Combination contraceptives: effects on weight. Cochrane Database of Systematic Reviews, (1).

Glintborg, D. (2016). Endocrine and metabolic characteristics in polycystic ovary syndrome. Danish Medical Journal. Apr;63(4):B5232.

Guastella, E., Longo, R. A., \& Carmina, E. (2010). Clinical and endocrine characteristics of the main polycystic ovary syndrome phenotypes. Fertility and sterility, 94(6), 2197-2201.

Hong, S. H., Sung, Y. A., Hong, Y. S., Jeong, K., Chung, H., \& Lee, H. (2017). Polycystic ovary morphology is associated with insulin resistance in women with polycystic ovary syndrome. Clinical endocrinology, 87(4), 375-380.

Jeanes, Y. M., \& Reeves, S. (2017). Metabolic consequences of obesity and insulin resistance in polycystic ovary syndrome: diagnostic and methodological challenges. Nutrition research reviews, 30(1), 97-105.

Lopes, I. M. R. S., Baracat, M. C. P., Simões, M. J., Simões, R. S., Baracat, E. C., \& Soares Jr, J. M. (2011). Endométrio na janela de implantação em mulheres com síndrome dos ovários policísticos. Revista da Associação Médica Brasileira, 57(6), 702-709.

Lopez, L. M., Grimes, D. A., \& Schulz, K. F. (2014). Steroidal contraceptives: effect on carbohydrate metabolism in women without diabetes mellitus. Cochrane Database of Systematic Reviews, (4).

Medeiros, SF (2017). Riscos, tamanho dos benefícios e implicações clínicas do uso de anticoncepcionais orais combinados em mulheres com síndrome dos ovários policísticos. Reproductive Biology and Endocrinology , 15 (1), 1-17.

Melo, A. S., Dos Reis, R. M., Ferriani, R. A., \& Vieira, C. S. (2017). Hormonal contraception in women with polycystic ovary syndrome: choices, challenges, and noncontraceptive benefits. Open access journal of contraception, $8,13$. 